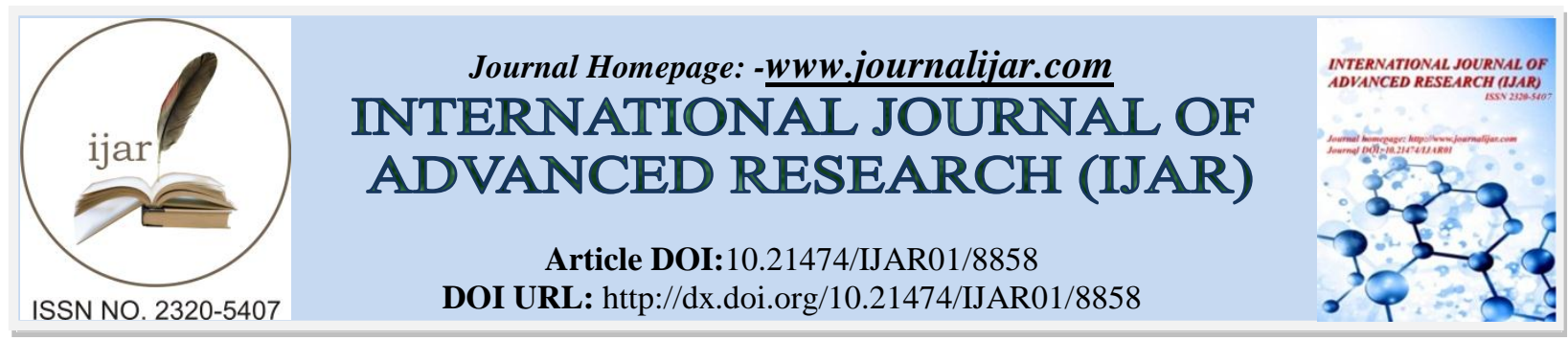

RESEARCH ARTICLE

\title{
CLINICAL AND ANGIOGRAPHIC PROFILE OF MYOCARDIAL INFARCTION WITH NONOBSTRUCTIVE CORONARY ARTERIES (MINOCA).
}

\author{
Routray S. N. ${ }^{1}$, Tripathy S. K. ${ }^{2}$, Satpathy C. ${ }^{3}$, Mohanty N.K. ${ }^{3}$ and Dash B.K. ${ }^{4}$ \\ 1. Professor, Dept. of Cardiology, S.C.B.Medical College. Cuttack, Odisha, India. \\ 2. Senior Resident, Dept. of Cardiology, S.C.B.Medical College. Cuttack, Odisha, India. \\ 3. Asso. Professor, Dept. of Cardiology, S.C.B.Medical College. Cuttack, Odisha, India. \\ 4. Asst.Professor, Dept. of Cardiology, S.C.B.Medical College. Cuttack, Odisha, India.
}

\section{Manuscript Info}

(.........................

Manuscript History

Received: 10 February 2019

Final Accepted: 12 March 2019

Published: April 2019

Key words:-

MINOCA, MICAD, dyslipidemia.

\section{Abstract}

Background: Myocardial Infarction with Non-Obstructive Coronary Arteries (MINOCA) remains a challenge in the clinical practice. Objective: This study has been designed to evaluate the prevalence, risk factors, clinical and angiographic profile of patients with MINOCA.

Methods: Two seventy four consecutive patients with Acute Myocardial infarction got admitted during the period Between March 2017- February 2019 were included in the study .They were classified in to MINOCA and MICAD group and their demographic, clinical, laboratory parameters, and angiographic features were compared. Result: Twenty-Five of 274 patients admitted for acute myocardial infarction (MI) were classified as MINOCA (prevalence 9.1\%). Patients with MINOCA were younger (46.1 vs 52.4 years, p <0.001) and the proportion of women was higher $(42.5 \%$ vs $18.7 \%, \mathrm{p}=0.03$ ) than the obstructive group. The MINOCA group had less cardiovascular risk factors (Diabetes $19.3 \%$ vs $31.1 \%$, Hypertension $17.3 \%$ vs $37.7 \%$ and smoking $45.3 \%$ vs $61.3 \%$ ). The presence of ST elevation MI was less (23.0\% vs $47.9 \%$ ) among MINOCA group. The prevalence of risk factors like dyslipidemia (36\% vs $38.7 \%$ ), obesity $(8.0 \%$ vs $9.5 \%)$, family history of CAD $(8.0 \%$ vs $8.0 \%)$ and presence of peripheral artery disease $(4.0 \%$ vs $1.0 \%)$ were similar in both the groups. Angiographic findings among the MINOCA group were recanalised and minimal $\mathrm{CAD}$ in $50.6 \%$, normal coronaries in $24 \%$, slow flow in $12 \%$, coronary spasm in $5.3 \%$. Myocardial bridging, coronary anomalies and coronary thrombus contributed $2.7 \%$ each.

Conclusions: MINOCA was more common in younger age group, females and patients with NSTEMI. Cardiovascular risk factors like Diabetes, Hypertension and smoking were less prevalent in MINOCA group and risk factors like dyslipidemia, obesity, family history of CAD and presence of Peripheral artery disease were similar in both the groups. In coronary angiography Recanalised and minimally obstructive lesion was found to be the commonest one followed by normal coronaries in MINOCA patients.

Corresponding Author:-Routray S. N.

Address:-Professor, Dept. of Cardiology, S.C.B.Medical College. Cuttack, Odisha, India. 


\section{Introduction:-}

Although the occurrence of an acute myocardial infarction without significant coronary artery disease (CAD) was initially reported almost 80 years ago, ${ }^{1}$ the term MINOCA (myocardial infarction with nonobstructive coronary arteries) has been used only recently to describe these patients. ${ }^{2}$ In keeping with the definition of MINOCA outlined in the 2016 European Society of Cardiology position paper, ${ }^{3}$ the term MINOCA should be reserved for those patients with an AMI (as defined by the "Third Universal Definition of Myocardial Infarction" 4 ) in the absence of obstructive coronary arteries and no other clinical findings to suggest alternative causes for the elevated cardiac biomarkers.

Coronary angiography may identify normal coronaries or minimal disease in patients with MINOCA. 'Significant' or ' obstructive CAD' or 'MICAD ' is defined by lesion > 50\% stenosis. There are various modifiable and non modifiable risk factors of acute myocardial infarction like diabetes, hypertension, dyslipidemia ,obesity, physical inactivity, age, family history of myocardial infarction, smoking and psychosocial stress.

This study has been designed to evaluate the prevalence, risk factors, clinical and angiographic profie in patients of acute myocardial infarction with non-obstructive coronary arteries (MINOCA).

\section{Material and Methods:-}

This is a single center observational Cross sectional study conducted during the period Between March 2017February 2019. Two seventy four consecutive patients with Acute Myocardial infarction (ST elevation Myocardial Infarction and Non ST elevation Myocardial Infarction ) got admitted in the Department of Cardiology, S.C.B. Medical College, Cuttack, India were included in the study. The study was approved by the institutional Ethical Committee. An Informed consent was obtained from all participants.

\section{Inclusion Criteria}

1. Evidence of Myocardial Infarction by significant elevation of a cardiac biomarker.

2. At least 2 of the following - ischemic symptoms, new ST/T changes, or new LBBB.

3. Qualitative coronary angiography findings to allow determination of the presence or absence of obstructive CAD.

\section{Exclusion Criteria}

1. Angiography was not performed in the context of an MI admission.

2. Prior history of significant coronary artery stenosis.

3. Hemodynamically unstable patients.

\section{Methods:-}

Relevant socio demographic data (age, gender), risk factors ( smoking, obesity, diabetes mellitus, systemic hypertension, dyslipidemia ,family history of premature CAD, and history of peripheral arterial disease), laboratory data(Blood sugar, Blood Urea, serum Creatinine, Lipid profile, Cardiac biomarkers ) were collected from patients and recorded in a proforma. Standard 12 leads ECG was recorded in all patients . Echocardiogram was done by PHILIPS CX 50 probe frequency $3.5 \mathrm{MHz}$ to analysis of regional wall motion abnormalities and assessment of LV systolic function and diastolic dysfunction and mechanical complications associated with MI. Coronary angiogram was done by SIEMENS Artis Zee Floor equipment, right femoral and radial artery route by modified Seldinger technique. Right and left coronary angiogram was done in various orthogonal view by using right and left Judkins and tiger diagnostic catheters. Angiogram was recorded in 15 frames/seconds.

\section{Statistical analysis}

Statistical analysis was done using IBM SPSS software. Continuous variables are presented as mean \pm SD. Categorical values are presented as percentages or proportion of whole. Student t test was used to compare difference in distribution of continuous variables. Chi Square test was used to detect significant difference between categorical variables. $\mathrm{P}$ value of $<0.05$ was taken as significant. Multivariate analysis was done using Logistic regression. Those variables which had possible association based on univariate analysis were considered for multivariate analysis. Backward conditional elimination was used in the multivariate model to eliminate those 
variables which did not have significant association. Final result of the multivariate model was computed in terms of Odds ratio and its corresponding $\mathrm{p}$ value.

\section{Results:}

Twenty-Five of 274 patients admitted for acute myocardial infarction (MI) were classified as MINOCA (prevalence 9.1\%). Patients with MINOCA were younger (46.1 vs 52.4 years, $\mathrm{p}<0.001)$ and the proportion of women was higher $(42.5 \%$ vs $18.7 \%, \mathrm{p}=0.03)$ than the obstructive group. The presence of STEMI was less $(23.0 \%$ vs $47.9 \%)$ among MINOCA group. The prevalence of risk factors like Diabetes (19.3\% vs 31.1\%), Hypertension (17.3\% vs $37.7 \%$ ) and smoking (45.3\% vs $61.3 \%$ ) were less in MINOCA group than MICAD group. The prevalence of risk factors like dyslipidemia (36\% vs $38.7 \%$ ), obesity $(8.0 \%$ vs $9.5 \%)$, family history of CAD $(8.0 \%$ vs $8.0 \%)$ and presence of peripheral artery disease $(4.0 \%$ vs $1.0 \%)$ were similar in both the groups (Table: 1$)$.

Table 1:-Baseline characteristics of patients with Myocardial Infarction

\begin{tabular}{|l|l|l|l|}
\hline Risk Factors & MINOCA & MICAD & P Value \\
\hline Mean Age (years) & 46.1 & 52.4 & $<0.001$ \\
\hline Female Sex & $42.5 \%$ & $18.7 \%$ & 0.03 \\
\hline smoker & $45.3 \%$ & $61.3 \%$ & 0.017 \\
\hline Diabetes & $19.3 \%$ & $31.1 \%$ & 0.026 \\
\hline HTN & $17.3 \%$ & $37.7 \%$ & 0.029 \\
\hline Dyslipidemia & $36 \%$ & $38.7 \%$ & 0.859 \\
\hline Obesity & $8.0 \%$ & $9.5 \%$ & 0.692 \\
\hline Family history of CAD & $8.0 \%$ & $8.0 \%$ & 0.999 \\
\hline Presence of PAD & $4.0 \%$ & $1.0 \%$ & 0.768 \\
\hline STEMI & $23.0 \%$ & $77 \%$ & 0.001 \\
\hline NSTEMI & $47.9 \%$ & 52.1 & \\
\hline
\end{tabular}

Figure 1:-Angiographic distribution of MINOCA

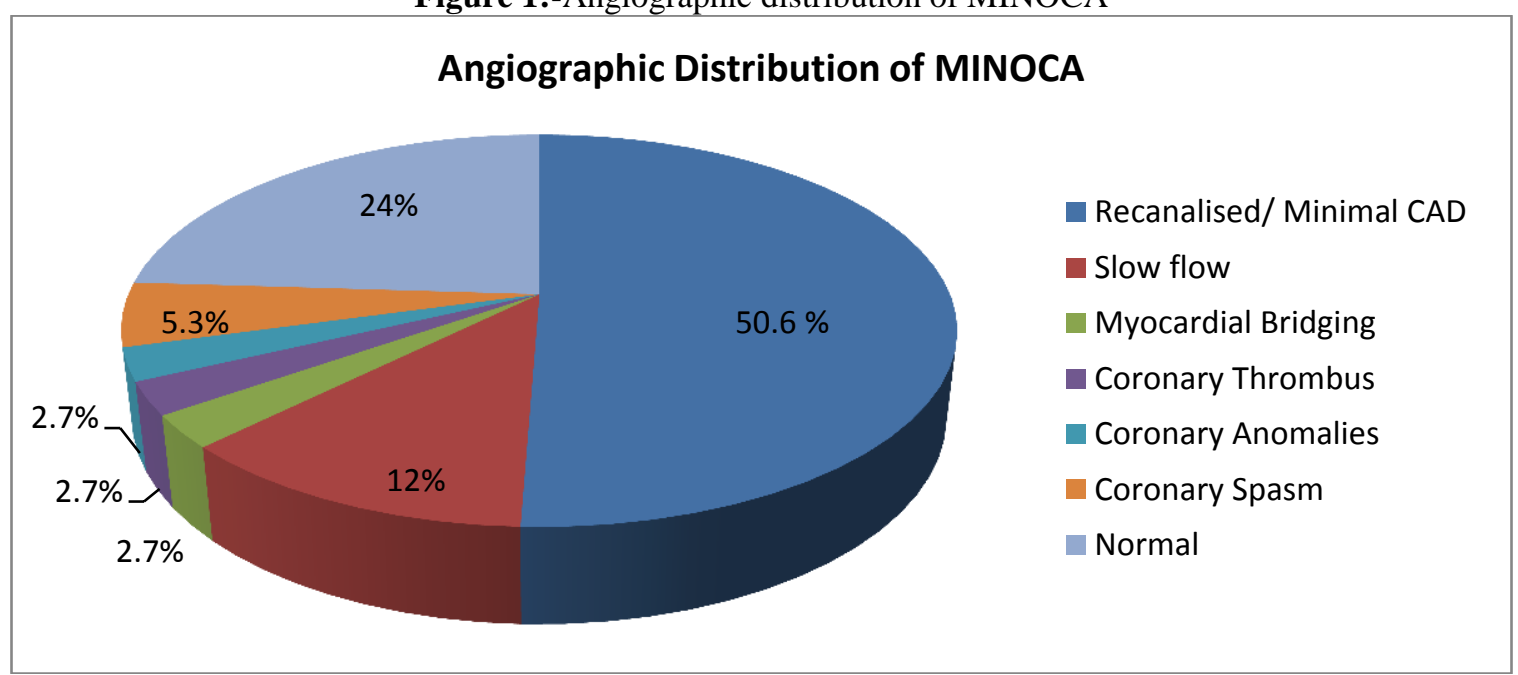


Figure 2:-Angiographic Distribution of MICAD

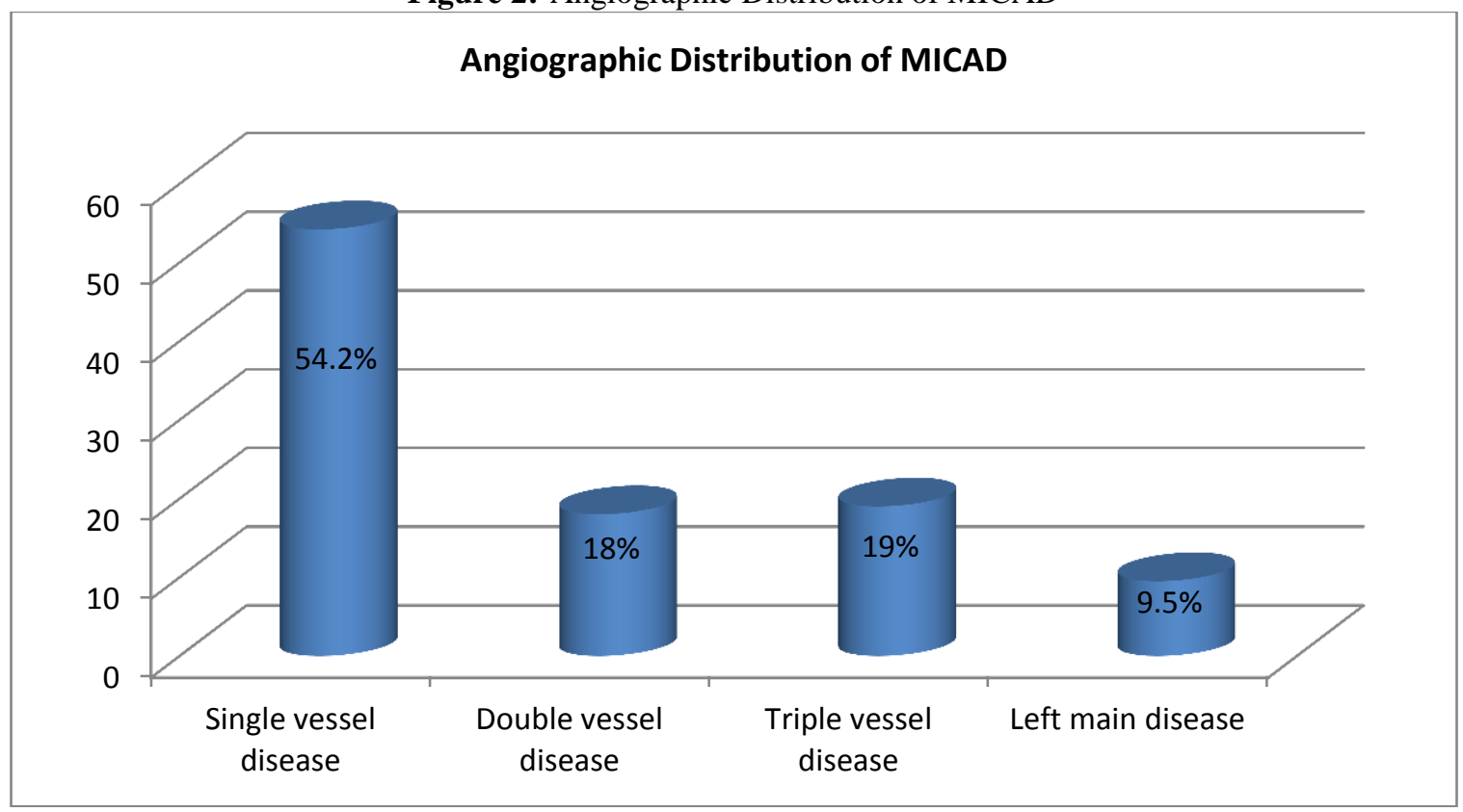

Angiographic findings among the MINOCA group were recanalised and minimal CAD was present in 50.6\% of patients, normal coronaries in $24 \%$, slow flow in $12 \%$ and coronary spasm in $5.3 \%$ of study population. Myocardial bridging, coronary anomalies and coronary thrombus contributed $2.7 \%$ each.

Among the obstructive CAD group Single vessel disease dominated the picture by about $54.2 \%$ followed triple vessel disease $19 \%$ and double vessel disease $18.0 \%$ and left main disease was present in $9.5 \%$.

\section{Discussion:-}

The diagnosis of MINOCA requires: (1) documentation of a acute myocardial infarct, (2) exclusion of obstructive CAD (3) no overt specific cause for the AMI presentation ${ }^{5,6}$. The diagnosis is made usually following invasive coronary angiography. Obstructive CAD is defined as an epicardial coronary artery stenosis of $\geq 50 \%$ on angiography $^{7}$. Hence, a stenosis $<50 \%$ is required for the diagnosis of MINOCA ${ }^{8,9}$.

In our study, twenty-Five of 274 patients (9.1\%) admitted for acute myocardial infarction (MI) were classified as MINOCA. It was $8 \%$ in study by Javier Lopez Pais et al ${ }^{10}$ and $10.8 \%$ in a study by Montenegro Sa Fet al ${ }^{11}$.

The mean age for MINOCA and obstructive CAD in our study was 46.1, 52.4 years respectively. According to Pasupathy $\mathrm{S}$ et $\mathrm{al}^{5}$, the mean age was $58.8,61.3$ years and according to Iqbal $\mathrm{M} \mathrm{N}$ et al ${ }^{12} 54,58$ years for MINOCA and obstructive CAD respectively. MINOCA is more common in younger age group. In our study patients with MINOCA and Obstructive CAD had younger age of presentation than other studies.

Among the total study population, $83.6 \%$ were males and $16.4 \%$ were females. The ST elevation MI was $86.5 \%$ in males and $62.2 \%$ in females. NSTEMI was $13.5 \%$ in males and $37.8 \%$ in females. According to our study females had higher NSTEMI. Females had a higher rate of MINOCA than obstructive CAD. In our study, it was $42.5 \%$ vs $18.7 \%$ for MINOCA and MICAD respectively. In Pasupathy S et $\mathrm{al}^{5}$ and Iqbal M N et al ${ }^{12}$ it was $43 \%, 43.8 \%$ for MINOCA and 24\%, $15.8 \%$ for MICAD.

In our study MINOCA patients were less likely to have hypertension (17.3\% vs 37.7\%) than obstructive CAD. Study by Pasupathy S et $\mathrm{al}^{5}$ showed (52\% vs $45 \%$ ), Iqbal M N et al ${ }^{12} 69.8 \%$ vs $\left.76.5 \%\right)$, Ramnath V S et al ${ }^{13}(61.5 \%$ vs $68.4 \%$ ) for MINOCA and obstructive CAD respectively. 
In our study, prevalence of diabetes was $19.3 \%$ in MINOCA and 31.1\% in MICAD. Study by Pasupathy S et al ${ }^{5}$ showed $15 \%$ vs $22 \%$, Iqbal M N et al ${ }^{12} 34.4 \%$ vs $39 \%$, Ramnath V S et al ${ }^{13} 23.6 \%$ versus $28.1 \%$ in MINOCA and obstructive CAD respectively. So prevalence of diabetes is less common in MINOCA group.

Prevalence of smoking was lesser in MINOCA than obstructive CAD (45.3\% vs 61.3\%) In our study. In other study, it was $42 \%$ vs $39 \%$ by Pasupathy S et $\mathrm{al}^{5}, 5.4 \%$ vs $18.4 \%$ by Iqbal M N et al ${ }^{12}, 19 \%$ vs $8.7 \%$ Ramnath V S et al ${ }^{13}$ in MINOCA and Obstructive CAD respectively. Overall prevalence of smoking was higher in our study population compared to the other studies.

Compared with MICAD patients, those with MINOCA also had closely similar percentage of dyslipidemia $38.7 \%$ vs $36 \%$. But the study by Pasupathy S et $\mathrm{al}^{5}$ showed $32 \%$ vs $21 \%$, Iqbal M N et al ${ }^{12} 45.3 \%$ vs $38.5 \%$, Ramnath V S et $\mathrm{al}^{13} 64.3 \%$ vs $48.4 \%$ dyslipidemia in MICAD and MINOCA respectively.

In both MINOCA and obstructive CAD group the family history of CAD was $8 \%$ in our study. Family history was positive in $21 \%$ vs $27 \%$ by pasupathi $\mathrm{S}$ et $\mathrm{al}^{5}, 28.1 \%$ vs $24.6 \%$ by Iqbal $\mathrm{M} \mathrm{N}$ et al ${ }^{12}$ and $45.3 \%$ vs $49.8 \%$ by Ramnath V S et $\mathrm{al}^{13}$ for MINOCA and obstructive CAD.

In our study, obesity was present in $8 \%$ vs $9.5 \%$ patients with MINOCA and obstructive CAD respectively. In Iqbal $\mathrm{M} \mathrm{Net} \mathrm{al}^{12}$ study prevalence of obesity was $26.9 \%$ vs $26.6 \%$ in MINOCA and MICAD group.

Compared to our study (4.0\% vs $1.0 \%)$, study by Ramnath V S et $\mathrm{al}^{13}$ (6.6\% vs $11.9 \%$ ) was associated with higher prevalence of peripheral arterial disease in patients with MINOCA and obstructive CAD respectively.

In our study among the MINOCA patients, 50.6\% had recanalised vessel with non obstructive lesion, 24\% had normal coronaries and $12 \%$ had slow flow. There is equal distribution of about $2.7 \%$ for Coronary anomalies, myocardial bridging and coronary thrombus each. In a study by Javier Lopez Pais et al ${ }^{10}$ the most prevalent pathophysiological mechanisms of MINOCA were plaque disruption (25\%) and stress myocardiopathy (25\%). Other mechanisms were coronary spasm (13.6\%), coronary embolus $(9.1 \%)$ and coronary artery dissection (2.3\%). In $11.4 \%$ of the patients they did not find the mechanism.

\section{Limitation}

The disparity in the value obtained in this study when compared to the other studies is mainly because of the ethnic and racial differences in various regions. The differences in values can be also attributed to the different methods and materials used in assessing the patients.

In addition to the conventional coronary angiogram, other imaging modalities like CMR, IVUS and OCT is needed to identify the potential pathological mechanism involved in this disorder. Due to financial constraint additional imaging modalities were not done in our study.

\section{Conclusion:-}

In our study, MINOCA was more common in younger age group, females and patients with NSTEMI. Cardiovascular risk factors like Diabetes, Hypertension and smoking were less prevalent in MINOCA group and risk factors like dyslipidemia, obesity, family history of CAD and presence of PAD were similar in both the groups. In coronary angiography Recanalised and minimally obstructive lesion was found to be the commonest one followed by normal coronaries in MINOCA patients.

However, it could be a chance finding due to small sample size, and it needs to be substantiated by a large ongitudinal study, so that it will help to reduce morbidity and improve quality of life.

\section{Conflict of Interest -}

Nil

\section{Financial support-}

Nil 


\section{Bibilography:-}

1. Gross H, Sternberg WH. Myocardial infarction without significant lesions of coronary arteries. Arch Intern Med. 1939;64:249-267.

2. Beltrame JF. Assessing patients with myocardial infarction and non-obstructed coronary arteries (MINOCA). J Intern Med. 2013;273:182-185.

3. Agewall S, Beltrame JF, Reynolds HR,et al; ESC working group position paper on myocardial infarction with non-obstructive coronary arteries. Eur Heart J. 2017;38:143-153.

4. Thygesen K, Alpert JS, Jaffe AS, et al. Third universal definition of myocardial infarction. J Am Coll Cardiol.2012;60:1581-1598.

5. Pasupathy $\mathrm{S}$ et al, Systematic Review of Patients Presenting With Suspected Myocardial Infarction and Nonobstructive Coronary Arteries. Circulation. 2015;131:861-870.

6. Pasupathy S, Tavella $\mathrm{R}$ et aI, The What, When, Who, Why, How and Where of Myocardial Infarction With Non-Obstructive Coronary Arteries (MINOCA), Circulation Journal. 2016; 80:11-16.

7. Beigel $\mathrm{R}$ et aI, Prognostic Implications of Nonobstructive Coronary Artery Disease $111 \quad$ Patients Undergoing Coronary Computed. Tomographic Angiography for Acute Chest Pain, Am J Cardiol 2013; $111: 941-945$

8. Beigel R, Sella Brosh $\mathrm{S}$ et al, The significance of non-obstructive coronary artery disease upon multi-detector computed tomography in patients presenting with acute chest pain, JACC 2012; 59(13): 13671367.

9. Segev A, Beigel $\mathrm{R}$ et aI, Non-obstructive coronary artery disease upon multi-detector computed tomography in patients presenting with acute chest pain-Results of an intermediate term follow-up, European Heart Journal of Cardiovascular Imaging 2012; 13: 169-173.

10. Javier Lopez Pais, Barbara Izquierdo et al,incidence, clinical profile and prognosis of patients with myocardial infarction with non-obstructive coronary arteries in the real world, JACC Mar 2017,69(11 Supplement) 142; DOI: 10.1016/S0735-1097(17)33531-3

11. 11. Montenegro Sa F, Ruivo $\mathrm{C}$ et al ,Myocardial infarction with nonobstructive coronary arteries: a single-center retrospective study, Coron Artery Dis. 2018 Sep;29(6):511-515.

12. Iqbal MN, Rao $\mathrm{M}$, et al. Non obstructive coronary artery disease in patients admitted for elective coronary angiography. Pak J Med Sci 2014;30(6): 1336-1340.

13. Ramanath V S, Armstrong D F et aI, Receipt of Cardiac Medications Upon Discharge Among Men and Women With Acute Coronary Syndrome. And Nonobstructive Coronary Artery Disease, Clin. Cardiol.2010; 33, 1 :36-41. 